\title{
Procesos de innovación en las organizaciones de salud y educación en Venezuela
}

\author{
D’Alvano, Luigi* \\ Hidalgo, Antonio**
}

\section{Resumen}

En la medida que crece la importancia de las organizaciones de servicio en las economías, y sus resultados son cada vez más determinantes en la calidad de vida de la población, aumenta el interés por estudiar sus procesos de innovación. El objetivo de este trabajo es analizar los procesos de innovación en un conjunto de organizaciones de servicio pertenecientes a los sectores de educación y salud en Venezuela. La investigación fue de tipo descriptivo con diseño no experimental transversal. Para el estudio se selecciono el modelo Temaguide, se propone el índice de desarrollo de los procesos de innovación y se aplicó una encuesta. Las organizaciones estudiadas presentan procesos de innovación "próximo a estructurado", con las fases en un nivel medio de desarrollo, siendo necesario vigilar la fase menos desarrollada. Se concluye que la trayectoria de desarrollo de las fases de los procesos de innovación está relacionada con las barreras y los recursos disponibles, y se plantea mejorar el alineamiento de los procesos de innovación con las tendencias del entorno, en una línea de investigación, desarrollo e innovación más orientada por las necesidades de los consumidores, proveedores y la sociedad.

Palabras clave: Innovación, salud, educación, modelos de innovación.

Recibido: 27-01-11 Aceptado: 09-10-11

* Máster en Administración de Empresas del Instituto de Estudios Superiores de Administración (IESA). Programa de Doctorado en Economía y Gestión de la Innovación, Universidad Politécnica de Madrid. Profesor de Gerencia de Innovación en Servicios del Instituto de Estudios Superiores de Administración (IESA). e-mail: Idalvano@reacciun.ve o Idalvano@gmail.com

** Catedrático y Director del Grupo de Investigación en Innovación, Propiedad Intelectual y Política Tecnológica (INNOPRO), y Director del Programa de Doctorado en Economía y Gestión de la Innovación. Universidad Politécnica de Madrid. e-mail: ahidalgo@etsii.upm.es 


\title{
Innovation Processes in Health and Education Organizations in Venezuela
}

\begin{abstract}
To the extent that the importance of service organizations in economies grows and their results become increasingly crucial to the population's quality of life, interest in studying innovation processes increases. The objective of this work is to study innovation processes in a group of service organizations belonging to health and education sectors in Venezuela. Research was of the descriptive type with a transversal, non-experimental design. The Temaguide model was selected for the study; an index for the development of innovation processes was proposed and a survey was applied. The organizations studied evidenced "close to structured" innovation processes with phases at a medium development level, monitoring the least developed phase. Conclusions were that the development trajectory for innovation process phases is related to barriers and available resources, and the study proposes improving the alignment of innovation processes with trends in the environment, in a line for research, development and innovation that is guided more by needs of the consumers, suppliers and society.
\end{abstract}

key words: Innovation, health, education, innovation models.

\section{Introducción}

La demanda creciente por parte de la sociedad para el desarrollo de servicios de calidad ha seguido al crecimiento experimentado por el sector, cada vez más importante en términos de empleo, actividad económica e impacto en la calidad de vida de la población. En este sentido, la importancia que los servicios han cobrado en la sociedad los está convirtiendo en un elemento clave y diferenciador para la competitividad de las organizaciones, incluidas las del sector manufactura (Robinson et al, 2002).

Cada día existe mayor interés por entender cómo la innovación puede contribuir a mejorar el funcionamiento de las organizaciones de servicio. En la medida que las tecnologías de comunicación e información aumentan la disponibilidad del conocimiento y el ciclo de vida de los productos y servicios se acorta (Chen y Xie,
2005), las organizaciones de servicio se encuentran en la necesidad de innovar sus conceptos, procesos, estructura organizacional y relación con la sociedad, con la finalidad de satisfacer las demandas crecientes de calidad, excelencia y bienestar exigidas por los consumidores (Christensen, 1997; Liikanen, 2003).

En el ámbito académico, el estudio de los procesos de innovación se ha convertido en un tema de suma importancia. La investigación de las dimensiones que conforman la innovación en servicio, sus características particulares en comparación con la innovación en el sector de manufactura y el desarrollo de modelos teóricos para el estudio del proceso de innovación son áreas de creciente desarrollo académico, así como los trabajos en investigación empírica relacionados con su validez y aplicación en el mundo organizacional. En este sentido, durante la Segunda Conferencia Anual sobre Innovación en Servicios y Productos (Karniou- 
Procesos de Innovación en las Organizaciones de Salud y Educación D’Alvano, Luigi y Hidalgo, Antonio

china et al, 2005), el desarrollo de nuevos servicios y sus procesos fue considerado uno de los temas de investigación de mayor interés para los académicos y los miembros de los cuerpos editoriales de publicaciones de investigación provenientes de las áreas de mercadeo y operaciones.

La importancia del sector servicios se pone también de manifiesto cuando se evalúa su participación en el producto interno bruto (PIB) de las naciones desarrolladas. Según el Banco Mundial (World Bank, 2010), para el año 2008 el 71.8\% del PIB de la zona Euro y más del $75 \%$ del PIB de los Estados Unidos correspondió al sector servicios. En Venezuela, según cifras del Banco Central (2010), el sector de servicios creció un $21 \%$ como proporción de la economía nacional en el período 1997-2009, representando para este último año el $54.2 \%$ del PIB. Siendo un país petrolero, en proceso de desarrollo, con una población en crecimiento, la demanda por más y mejores servicios de salud y educación, entre otros, son parte fundamental de su agenda de desarrollo económico y social.

El objetivo de este trabajo es analizar los procesos de innovación en un conjunto de organizaciones de servicio pertenecientes a los sectores de educación y salud en Venezuela, prestando particular interés en conocer cómo la trayectoria del desarrollo del proceso de innovación se relaciona con las barreras y los recursos disponibles para la organización.

La investigación fue de tipo descriptivo con diseño no experimental transversal (Hernández Sampieri et al., 1997). El desarrollo de la investigación se inició con la revisión teórica en el área de innovación en servicios, que permitió definir el modelo empleado en el estudio. El modelo seleccionado incluye una encuesta basada en la definición de cada una de las fases del proceso de innovación (Fundación para la Innovación Tecnológica -COTEC-, 2004: 147-149) con una escala de tres posibles estadíos: informal, medio o avanzado; a la que adicionalmente se incorporó una pregunta sobre las barreras o limitaciones bajo las cuales la organización desarrolla sus proyectos de innovación.

La encuesta fue aplicada a organizaciones de servicio venezolanas pertenecientes a los sectores salud (instituciones privadas clasificadas como tipo "A" según la norma COVENIN 2339-87) y educación (universidades que tuvieran carreras, programas y/o escuelas de ingeniería dentro de su oferta académica de pregrado). El instrumento fue enviado aleatoriamente obteniéndose respuesta de 8 organizaciones de salud de un universo total de 16 clínicas, y 10 universidades de un total de 45 instituciones. El trabajo de campo se realizó entre los meses de septiembre del año 2009 y febrero del año 2010.

\section{El concepto de servicio}

Según Gadrey (1992:19) “los servicios se definen como el conjunto de operaciones de procesamiento llevadas a cabo por el servidor $(B)$ en nombre de un cliente $(A)$, en un medio $(C)$ tomado por $A$, y destinado a provocar un cambio de estado del medio C". Otra definición de interés del mismo autor añade que "producir un servicio es organizar una solución a un problema que no implica suministrar un 
producto. Consiste en poner a disposición del cliente un conjunto de competencias para organizar una solución que puede ser proporcionada con distintos grados de precisión" (Gadrey et al., 1995:5).

En este trabajo se propone como servicio el conjunto de actividades enlazadas a través de actos de comunicación, en secuencias parciales o totales, que son realizadas de forma simultánea con los recursos y competencias del cliente y el servidor, donde participan los proveedores, para alterar el estado del cliente, o de un objeto o archivo de su interés o propiedad, con una finalidad, en un entorno cultural determinado.

Una serie de características propias de los servicios (Fitzsimmons y Fitzsimmons, 1998) determinan la naturaleza de su innovación. La intangibilidad, la interactividad y la coproducción son elementos fundamentales para el diseño y gerencia del proceso de servicio. La heterogeneidad se manifiesta en la diversidad de sectores, desde recreación hasta servicios intensivos en conocimiento, lo que impone la necesidad de desarrollar tanto conceptos de uso general, como investigar las peculiaridades de cada subsector servicio. La inapropiabilidad del servicio se traduce en la búsqueda de nuevas formas de protección de la propiedad intelectual y la caducidad impone estrategias gerenciales para mantener una alta tasa de ocupación de la capacidad instalada.

Más reciente, asociado al concepto de servicio, en la dimensión operacional se propone el concepto de sistema de servicio (IfM \& IBM, 2008:6). Este es definido "como una configuración dinámica de recursos que crea y entrega valor entre un servidor y el cliente a través del servicio. En este sentido, tiene su complejidad en la forma que dichos recursos interactúan, en general de manera no lineal". Bajo esta definición, el estudio de la innovación de las organizaciones de servicio trasciende el estudio de los proyectos de innovación y requiere el análisis del proceso de innovación a nivel organizacional.

\section{La innovación en servicios}

En sus estudios, Schumpeter (1934) argumenta cómo el desarrollo económico es conducido por la innovación a través de procesos dinámicos de destrucción creativa, en los cuales las nuevas tecnologías remplazan las existentes. Bajo esta visión, Schumpeter propuso las siguientes formas de innovación: (i) la introducción de un nuevo bien; (ii) la introducción de nuevas formas de producción; (iii) el descubrimiento de una nueva fuente de materias primas o producto semi-elaborado; (iv) la apertura de un nuevo mercado; y (v) la creación de nuevas estructuras de mercado en una industria.

Los primeros estudios en el área de innovación provienen del área de manufactura, con particular inclinación por el estudio de la innovación tecnológica. Freeman (1974) propone que la innovación requiere ser un proceso bajo control en lugar de arbitrario y casuístico, y que una parte importante de su manejo está relacionado a los aspectos económicos de la innovación, tales como, costo, retorno sobre la inversión, estructura de mercado, tasa de crecimiento y distribución de los posibles beneficios. Dentro de este ámbito, se define la innovación tecnológi- 
Procesos de Innovación en las Organizaciones de Salud y Educación D’Alvano, Luigi y Hidalgo, Antonio

ca "como el conjunto de las etapas técnicas, industriales y comerciales que conducen al lanzamiento con éxito en el mercado de nuevos productos y servicios o la utilización comercial de nuevos procesos técnicos" (Hidalgo et al, 2002:64).

Con el objetivo de incorporar y profundizar el estudio del sector servicios en las encuestas de innovación, el Manual de Oslo propone la siguiente definición (OCDE, 2006:56): "una innovación es la introducción de un nuevo, o significativamente mejorado producto (bien o servicio), de un proceso, de un nuevo método de comercialización o de un nuevo método organizativo, en las practicas internas de la empresa, la organización del lugar de trabajo o las relaciones exteriores". Esta definición abarca distintos tipos de innovación, incorporando aquellas de carácter no tecnológico más comunes en el sector servicios, tales como, la innovación en mercadotecnia y la innovación organizacional.

Dentro de este contexto, Toivonen y Touominen (2009: 893) enfatizan el sentido de la innovación en la creación de valor para el cliente y el servidor cuando proponen que la innovación en servicios es "un nuevo servicio o la mejora de un servicio existente que provee beneficios para la organización que la ha desarrollado, beneficio que usualmente deriva en el valor agregado que la innovación provee para los clientes".

Por su parte, Den Hertog et al. (2010) proponen que la innovación de servicios está asociada a cambios en las siguientes dimensiones: (i) el concepto de servicio; (ii) la interacción con el cliente; (iii) el sistema de valores, roles o socios de negocio; (iv) el nuevo modelo de ingresos; (v) el sistema de entrega del servicio desde la perspectiva organizacional; y (vi) el sistema de entrega de servicio basado en las nuevas opciones tecnológicas. El desarrollo de las tecnologías de información y comunicaciones, en particular internet, explica la necesidad de innovar en el modelo de ingresos o el sistema de valores, roles o socios de negocios. Precisamente en internet, por ejemplo en los medios de comunicación, sus innovaciones presentan el reto de desarrollar mecanismos que les permitan generar ingresos a partir de los nuevos servicios electrónicos, desarrollados a través de nuevos roles en el esquema de producción de contenidos. Además, la coproducción y la intangibilidad cada vez más determinan el concepto de innovación en servicios mediante el desarrollo de las dimensiones del concepto, la interacción y el sistema de entrega.

\section{Modelos para el estudio de la innovación}

Inicialmente los estudios de innovación tuvieron como objeto de investigación a los innovadores y sus proyectos. En la medida que la complejidad tecnológica u organizacional de los proyectos de innovación crece y se requiere de mayores recursos y competencias, ha crecido el interés por entender cómo el proceso de innovación se desarrolla a nivel organizacional, cuáles son las mejores prácticas, su trayectoria de desarrollo y sus oportunidades de mejora. En este sentido, se describen a continuación cuatro modelos desarrollados para el estudio de la innovación en el contexto de la organización. 
El primer modelo, denominado "Innovación basado en Procesos" (Chiesa et al, 1996), está dirigido a entender el proceso de innovación tecnológica en el sector de manufactura a partir del desempeño organizacional y el uso de sistemas y herramientas. Bajo el auspicio del Departamento de Industria y Comercio del Reino Unido, este modelo fue desarrollado como un instrumento para mejorar la competitividad de las organizaciones mediante un proceso de auditoría de innovación. El modelo persigue el estudio de la innovación basado en cuatro procesos medulares: (i) la identificación y generación de nuevos conceptos de productos; (ii) el desarrollo de los proyectos de innovación seleccionados; (iii) el desarrollo del sistema productivo asociado al nuevo producto; y (iv) la gerencia de la tecnología, incluida su adquisición. Relacionados y soportando la ejecución de los procesos medulares se encuentran tres procesos facilitadores: los recursos (humanos y financieros), los sistemas y herra- mientas, y el liderazgo. El modelo se detalla en el Diagrama 1.

La aplicabilidad del modelo se basa en su capacidad para auditar las prácticas y desempeños de innovación, determinando brechas, proyectos y planes de acción tendientes a mejorar la competitividad de la organización. Los autores sugieren que el proceso de auditoría de la innovación debe otorgar especial atención al proceso de diseño y desarrollo de producto, la organización y gerencia del grupo de proyecto, así como el lanzamiento acertado y una adecuada distribución de la innovación. Por otra parte, si bien este modelo de innovación está centrado en la innovación tecnológica, presenta las fortalezas derivadas del análisis de la innovación desde la perspectiva de los procesos y la aplicación de un procedimiento de auditoría que facilita la búsqueda de oportunidades a través del análisis de las mejores prácticas, pero requiere incorporar las particulares dimensiones no tecnológicas del servicio dentro

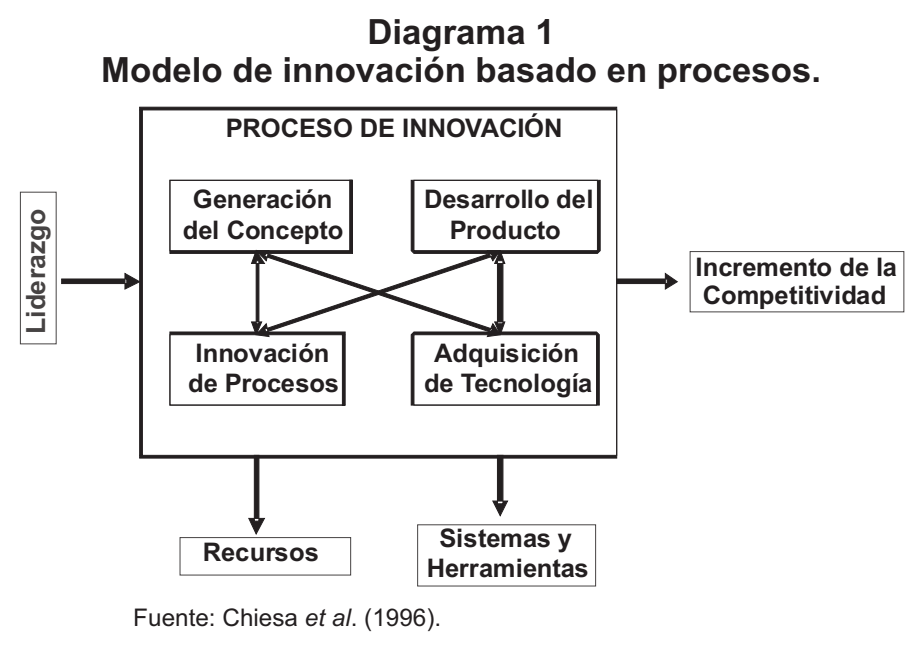


Procesos de Innovación en las Organizaciones de Salud y Educación D’Alvano, Luigi y Hidalgo, Antonio

de sus variables de análisis para ser aplicable a este tipo de organización.

El segundo modelo, conocido como "Pentatlón" (Goffin y Pfeiffer, 1999), constituye un marco genérico para el estudio de la innovación y ha sido desarrollado a partir de la investigación comparativa de las prácticas de gestión de los proyectos de innovación desarrollados por las organizaciones de manufactura en el Reino Unido y Alemania. Este modelo se compone de cinco elementos cuyo estudio permite identificar las prácticas de la gestión de la innovación y su interrelación: la estrategia de innovación, la gestión de la creatividad y las ideas, la selección y gestión del portafolio, la etapa de implementación, y la gestión de recursos humanos. El modelo se muestra en el Diagrama 2.

El modelo pentatlón está centrado en el análisis de la gestión del proyecto de innovación. La porción central del modelo se refiere a los procesos que desarrollan la innovación dentro de la organización industrial, conocido como el túnel de de- sarrollo de proyectos (Oke y Goffin, 2001). Este túnel ilustra las actividades claves del desarrollo de la innovación: generación de ideas, selección y desarrollo, implementación y lanzamiento en el mercado. Se asemeja a las fases planteadas por Sundbo (1997) para el desarrollo de un proyecto de innovación en servicios. Las secciones superior e inferior identifican los aspectos organizacionales que contribuyen a la creación del clima interno necesario para garantizar el adecuado desarrollo del proyecto de innovación. Ambas actividades son complementarias entre sí, pues el desarrollo y ejecución de los proyectos de innovación requiere contar con la estrategia y los recursos adecuados para su desarrollo y lanzamiento en el mercado.

La idea clave de este modelo es entender que las organizaciones requieren tener un buen desempeño en las cinco áreas para lograr un desarrollo satisfactorio de sus proyectos de innovación. Similar a la competencia de pentatlón, para ser un atleta competitivo se requiere de

\section{Diagrama 2}

Modelo pentatlón.

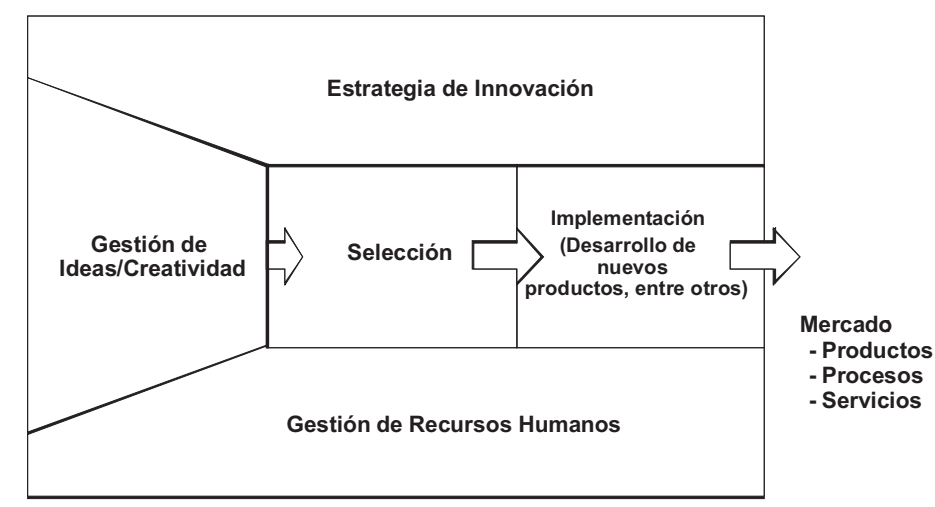

Fuente: Goffin, K. y Pfeiffer,R. (1999). 
un resultado exitoso en las cinco disciplinas. En este sentido, el modelo puede ser utilizado como una herramienta de diagnóstico tanto de cada área individual como del desarrollo general de cada proyecto de innovación.

Basado en los conceptos desarrollados para evaluar la gestión de la excelencia en el Premio Nacional de Calidad Malcolm Baldrige (NIST, 2006) y el Premio Europeo a la Excelencia (EFQM, 2003), entre otros, Arzola y Mejías (2007) proponen el "Modelo de las Siete Dimensiones (7D's)". Este modelo está centrado en el análisis de la gestión de las prácticas de innovación en las organizaciones de servicios y se basa en el estudio de las siguientes dimensiones: (i) liderazgo; (ii) planificación estratégica; (iii) competencias del recurso humano; (iv) procesos; (v) organización; (vi) satisfacción del cliente; y (vii) responsabilidad social. Cada una de las siete dimensiones ocurren de manera concurrente, interrelacionadas y atendiendo a las variables consideradas en su análisis. El modelo se muestra en el Diagrama 3.
Las dimensiones liderazgo y planificación estratégica se interrelacionan en vista que corresponden a la gestión de la organización, su visión y direccionamiento. Las dimensiones procesos, recursos humanos y organización se interrelacionan en vista que corresponden al análisis de los elementos del sistema de servicio. Las dimensiones satisfacción del cliente y responsabilidad social se interrelacionan en el nivel de resultados de la gestión de innovación y el desempeño de excelencia. Por último, la aplicación del modelo permite identificar oportunidades de mejora de la gestión de la innovación a partir del análisis de las siete dimensiones mencionadas y su comparación con las mejores prácticas. La naturaleza del análisis debería facilitar el desarrollo de un portafolio de proyectos de innovación orientado hacia el desempeño de excelencia.

Finalmente, se presenta el cuarto modelo, denominado "Temaguide", que por su enfoque de proceso desagregado en fases por grupo de actividades, ha sido aplicado con éxito en el estudio de

\section{Diagrama 3}

\section{Modelo para medir la innovación en el sector servicios.}

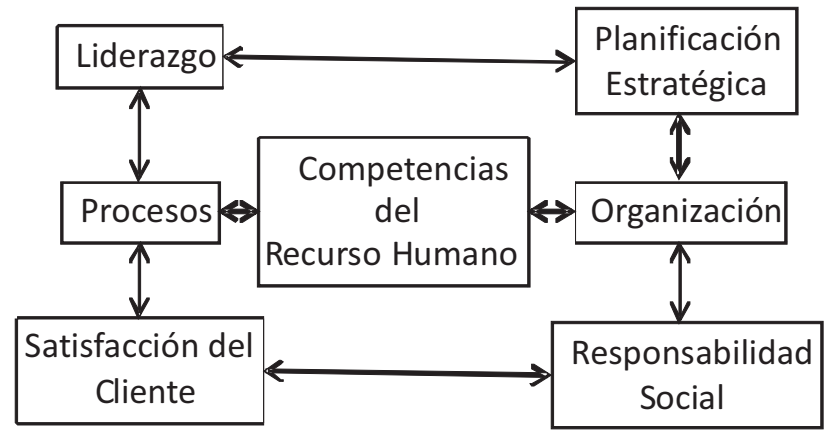

Fuente: Arzola, M. y Mejías, A. (2007) 
Procesos de Innovación en las Organizaciones de Salud y Educación D’Alvano, Luigi y Hidalgo, Antonio

los procesos de innovación en organizaciones de servicio (COTEC, 2004).

Este modelo (COTEC, 1999), mostrado en el Diagrama 4, es el resultado de un proyecto de investigación que fue apoyado por el Programa de Innovación desarrollado por la Dirección General XIII de la Comisión Europea y realizado por un grupo de organizaciones europeas: la Fundación para la Innovación Tecnológica COTEC (coordinadora del proyecto), SOCINTEC (empresa consultora), CENTRIM (Universidad de Brighton), IRIM (Universidad de Kiel) y la Unidad de Investigación y Desarrollo de la Escuela de Negocios de Manchester (Manchester Business School).

La ventaja de este modelo es que el elemento de análisis del proceso de innovación son las actividades realizadas en cada fase. El modelo permite evaluar el proceso de innovación, sin diferenciar si el proyecto que fluye por el túnel de innovación corresponde a una dimensión tecnológica o no tecnológica.

El proceso de innovación de la organización de servicio se analiza a través de las actividades realizadas en las siguientes cinco fases: vigilar, focalizar, capacitar, implantar y aprender, y se enmarca dentro de la filosofía de la organización que aprende.

El modelo se plantea como una herramienta para el estudio de la innovación en la organización, así como una metodología sencilla para gestionar el cambio, reforzar la gestión tecnológica y aumentar la base de conocimientos y experiencias de la organización en el desarrollo de sus proyectos de innovación.

Asimismo, la trayectoria de desarrollo del proceso de innovación se entiende como la secuencia con la cual la organización de servicio desarrolla las fases del proceso de innovación.

En la primera fase, denominada vigilar, la organización busca las señales sobre la necesidad de innovar, así como las oportunidades de mercado y tecnología, con el objeto de prepararse para afrontar los cambios que le puedan afectar en un futuro más o menos próximo. Esta actividad requiere el esfuerzo sistemático y organizado de observación, cap-

\section{Diagrama 4 \\ Modelo de innovación tecnológica Temaguide.}

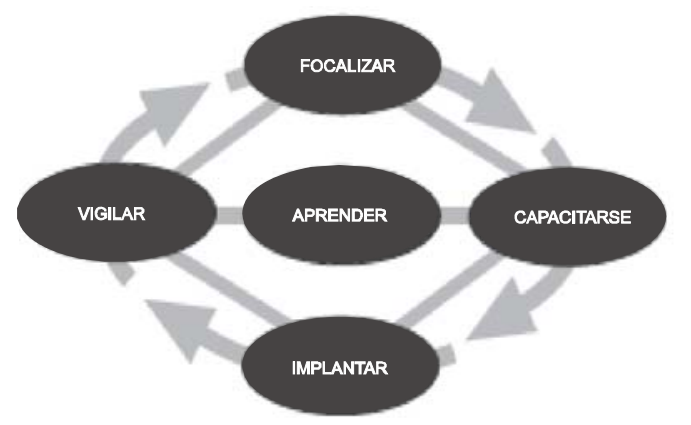

Fuente: Fundación COTEC (1999). 
tación, análisis, difusión precisa y recuperación de información sobre los hechos del entorno económico, social y tecnológico, relevantes para identificar una oportunidad o amenaza, con el objeto de poder tomar decisiones de menor riesgo en el momento oportuno (Palop y Vicente, 1999).

La segunda fase, denominada focalizar, agrupa el conjunto de actividades que trata de definir una respuesta estratégica a partir de la información recogida en la etapa anterior. La fase de focalizar consta de cuatro actividades: (i) la generación de ideas como oportunidades de innovación; (ii) la preselección de ideas sobre las innovaciones posibles; (iii) la selección de las innovaciones a estudiar; y (iv) el estudio, la selección final y aprobación de las innovaciones que pasarán a ser desarrolladas.

La tercera fase, denominada capacitar, abarca el conjunto de actividades encaminadas a dotar a la organización de las competencias (conocimientos, personas y equipos) necesarias para el correcto funcionamiento del proceso de innovación. Se trata de una fase que se desarrolla en dos ámbitos: proyectos y organizacional. En el ámbito del proyecto de innovación debe dotar a sus miembros de las herramientas necesarias para su desarrollo e implantación, y en el ámbito organizacional debe proveer las competencias necesarias para facilitar e impulsar el proyecto a un final exitoso. Entre las principales actividades de esta fase se encuentran la formación de personal, el establecimiento de alianzas, la contratación de personal calificado, los servicios de consultoría, la compra de equipos, el intercambio de información y conocimien- tos, los contratos de "know-how" y la subcontratación de proveedores.

La cuarta fase, denominada implantar, constituye el núcleo del proceso y es donde la innovación se va a hacer realidad. En esta fase es importante definir el equipo de proyecto, las responsabilidades y dedicación de sus integrantes. Además, se requiere contar con un proceso de trabajo definido, una buena gerencia de proyecto y habilidades de gestión del cambio. Los elementos claves de esta fase son la asignación de recursos, la prioridad e importancia que el liderazgo de la organización otorgue al proyecto y su adecuada administración.

Finalmente, el proceso de innovación contempla actividades relacionadas a su mejora en el tiempo. La quinta fase, denominada aprender, soporta el principio de que el proceso de innovación ha de contar también con una fase de retroalimentación que permita conocer su funcionamiento y mejorarlo mediante la recolección y almacenamiento de los conocimientos obtenidos en el desarrollo de los proyectos. La sistematización de la fase de aprender en el proceso de innovación permite a la organización la reflexión de sus éxitos y fracasos, así como documentar y trasmitir hacia el futuro el conocimiento producto del desarrollo de los proyectos de innovación ejecutados.

En esta fase, las organizaciones deben desarrollar diversas herramientas y prácticas que le permitan documentar, medir, evaluar, comparar y descubrir mejoras en su proceso de innovación.

En el estudio realizado por la Fundación para la Innovación Tecnológica COTEC (2004) en el sector servicios, se establece que cada fase tiene un peso di- 
Procesos de Innovación en las Organizaciones de Salud y Educación D’Alvano, Luigi y Hidalgo, Antonio

ferente a la hora de definir el grado de madurez del proceso de innovación, las fases capacitar y aprender son las que tienen mayor importancia, pues se considera que su desarrollo corresponde a la etapa más avanzada de desarrollo del proceso de innovación. De esta forma, las escalas de cada fase del modelo se integran en una escala general compuesta por cinco niveles: casual (nivel 1), informal (nivel 2), estructurado (nivel 3), integrado (nivel 4) y avanzado (nivel 5), que sirve como aproximación final del nivel de madurez del proceso de innovación en la organización, como se muestra en el Diagrama 5.

Para evaluar el grado de desarrollo de los procesos de innovación de las organizaciones analizadas se define el índice COTEC (IC) de desarrollo del proceso de innovación como la sumatoria de las valoraciones de cada una de las fases (Fi):

$$
\text { IC }=\sum_{i=1}^{5} \overline{F_{i}}
$$

De esta forma, el índice COTEC puede adquirir una calificación que oscila entre los 10 puntos como mínimo (nivel casual) y 20 puntos como máximo (nivel avanzado). Por último, la encuesta cierra con una pregunta que solicita al entrevistado la opinión general sobre el estado de la innovación en su organización.

\section{Procesos de innovación: sectores salud y educación}

En esta investigación se selecciono y aplico el modelo Temaguide porque tiene como objeto de estudio el proceso de innovación, su unidad de análisis son las actividades, presenta un instrumento de medición sencillo y su aplicación en España demostró su validez para describir el proceso de innovación en diferentes organizaciones del sector servicios (COTEC, 2004).

Para entender el contexto en el cual se desarrollan los procesos de innovación de las organizaciones estudiadas, la primera pregunta de la encuesta se refirió a las barreras a la innovación. Hay que destacar, que tanto las organizaciones de salud como de educación mencio-

\section{Diagrama 5 \\ Escala de medición del modelo.}

\begin{tabular}{|c|c|c|c|c|}
\hline Nivel1 & Nivel2 & Nivel 3 & Nivel4 & Nivel5 \\
\hline Casual & Informal & Estruturado & Integrado & Avanzado \\
\hline
\end{tabular}

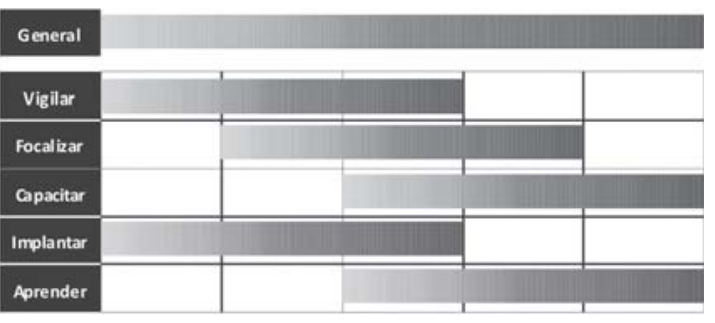

Fuente: Fundación COTEC (2004). 
naron el entorno económico poco favorable como la primera barrera a la innovación (Tabla 1).

Sin embargo, para las organizaciones de salud, la segunda barrera más mencionada es la existencia de costos elevados de innovación, mientras que para las organizaciones de educación es la falta de fuentes de financiamiento o limitaciones presupuestarias.

Los resultados del índice COTEC (IC) de desarrollo de los procesos de innovación de las organizaciones estudiadas se presentan en la Tabla 2. Se aprecia que ambas organizaciones presentan un valor del índice COTEC bastante similar (15.13 en salud y 14.10 en educación), lo que pone de relieve la existencia de un cierto grado de estructuración en el proceso de innovación y la adopción de algunas prácticas avanzadas de gestión. El análisis de ambos sectores a nivel desagregado, en función de las cinco fases del modelo, muestra simi- litud en los valores obtenidos para cuatro fases: focalizar, capacitar, implantar y aprender presentan un nivel de desarrollo medio, mientras la fase vigilar es la menos desarrollada presentando un nivel de desarrollo informal.

Dado que las fases dentro del modelo son evaluadas en escalas distintas, se ha optado por desarrollar un índice relativo que permita mostrar el nivel de oportunidad de desarrollo, en términos porcentuales, de cada una de las fases del proceso de innovación. Para ello se define el índice porcentual de oportunidad de desarrollo de la fase de innovación (IPODFI) como:

$I P O D F I=\frac{(\text { Valor superior escala }- \text { Valor obtenido })}{\text { Valor sup erior escala }- \text { Valor Inferior escala }}$

De acuerdo con este índice, la fase más desarrollada corresponde a aquella cuyo valor sea más bajo. Los resultados de la Tabla 3 apuntan a que en las organizaciones de salud, las fases más desa-

Tabla 1

Barreras a la innovación.

\begin{tabular}{lcc}
\hline \multicolumn{1}{c}{ Barreras a la Innovación } & \multicolumn{2}{c}{ Menciones } \\
\cline { 2 - 3 } & Salud & Educación \\
\hline Entorno económico poco favorable & 7 & 6 \\
Costos de innovación elevados & 6 & 4 \\
Falta de fuentes de financiamiento o presupuesto limitado & 1 & 6 \\
Cultura organizacional adversa al riesgo & 0 & 4 \\
Falta de información sobre los mercados & 1 & 2 \\
Estructura organizacional muy rígida y madura & 0 & 3 \\
Falta de personal calificado & 1 & 1 \\
Falta de información sobre tecnología & 1 & 1 \\
Mercado o sector de servicio protegido & 2 & 0 \\
\hline
\end{tabular}

Fuente: Elaboración propia (2011). 
Procesos de Innovación en las Organizaciones de Salud y Educación D’Alvano, Luigi y Hidalgo, Antonio

Tabla 2

Desarrollo de los procesos de innovación.

\begin{tabular}{lcccc}
\hline Fase & Salud & Educación & Evaluación & Escala \\
\hline Vigilar & 1.88 & 1.50 & Informal & $1-3$ \\
Focalizar & 3.13 & 2.90 & Medio & $2-4$ \\
Capacitar & 4.25 & 3.80 & Medio & $3-5$ \\
Implantar & 2.00 & 2.10 & Medio & $1-3$ \\
Aprender & 3.88 & 3.80 & Medio & $3-5$ \\
IC & $\mathbf{1 5 . 1 3}$ & $\mathbf{1 4 . 1 0}$ & Medio & $\mathbf{1 0 - 2 0}$ \\
\hline
\end{tabular}

Fuente: Elaboración propia (2011).

Tabla 3

Índice porcentual de oportunidad de desarrollo de la innovación.

\begin{tabular}{|c|c|c|}
\hline Fase & Salud & Educación \\
\hline Vigilar & $56,2 \%$ & $75,0 \%$ \\
\hline Focalizar & $43,7 \%$ & $55,0 \%$ \\
\hline Capacitar & $37,5 \%$ & $60,0 \%$ \\
\hline Implantar & $50,0 \%$ & $45,0 \%$ \\
\hline Aprender & $56,2 \%$ & $60,0 \%$ \\
\hline IPODFI & $48,7 \%$ & $59,0 \%$ \\
\hline
\end{tabular}

Fuente: Elaboración propia (2011).

rrolladas son capacitar $(37,5 \%)$ y focalizar $(43,7 \%)$, lo que se explica debido a que gran parte de las innovaciones en este sector están asociadas a la incorporación de equipos y conocimientos desarrollados por sus proveedores tecnológicos, los cuales difunden y promueven la incorporación de nuevas tecnologías, y realizan procesos de capacitación durante su implantación. Este resultado refleja la existencia de una trayectoria de innovación en estas organizaciones conocida como innovación orientada por los suplidores de tecnología (Den Hertog et al, 2003; Tidd et al, 2005). Sin embargo, para las organizaciones de educación la fase más desarrollada es implantar
$(45,0 \%)$, seguida de focalizar $(55,0 \%)$, lo que puede explicarse por el uso de las capacidades de gerencia de proyecto típicas de las escuelas de ingeniería, en un contexto de fuertes limitaciones presupuestarias. Este resultado refleja caracteristicas de la trayectoria de innovación conocida como de base científica (Tidd et al, 2005).

Por último, en la Tabla 4 se compara el índice COTEC (IC) de desarrollo de los procesos de innovación y el resultado de la pregunta final de la encuesta referida a la opinión general del proceso de innovación de la organización. Para comparar ambos resultados se presenta entre paréntesis el porcentaje equivalente de desarrollo del 
Tabla 4

IC y opinión general del proceso de innovación.

\begin{tabular}{ccc}
\hline Sector & Índice COTEC & Pregunta final encuesta \\
\hline Salud & $15.13(51 \%)$ & $3.13(56 \%)$ \\
Educación & $14.10(41 \%)$ & $2.80(40 \%)$ \\
\hline
\end{tabular}

Fuente: Elaboración propia (2011).

proceso de innovación que representa los valores obtenidos del índice COTEC (IC) y la respuesta de la pregunta final.

En las organizaciones de salud, la pregunta sobre la opinión general del proceso de innovación arrojó una evaluación final de 3.13 puntos sobre un máximo de 5 , equivalente a un $56 \%$ de la máxima calificación posible, mientras que el valor del índice COTEC (15.13 puntos) equivale a un $51 \%$ de la máxima calificación posible. Se observa que ambos porcentajes son cercanos y permiten corroborar la utilidad del índice COTEC propuesto. Por tanto, se puede afirmar que este valor del índice COTEC ubica el estado de desarrollo de la innovación en el nivel estructurado para las organizaciones de salud (clínicas y hospitales privados tipo "A"), lo que implica que estas organizaciones cuentan con un proceso de innovación parcialmente desarrollado, en particular las fases de capacitar y focalizar, tienen un responsable de cada proyecto, más la innovación no se desarrolla como parte de un proceso integrado a lo largo de la organización.

Por su parte, en las organizaciones de educación, la pregunta sobre la opinión general del proceso de innovación arrojó una evaluación final de 2.80 puntos, equivalente a un $40 \%$ de la máxima calificación, mientras que el valor del índice COTEC (14.10 puntos) equivale a un
$41 \%$ de la máxima calificación posible. Al igual que antes, se observa también que ambos porcentajes son cercanos y permiten corroborar la utilidad del índice Cotec propuesto, por lo que se puede afirmar que este valor del índice COTEC ubica el estado de desarrollo de la innovación en el nivel próximo a estructurado para las organizaciones de educación (universidades con carreras de ingeniería), con particular desarrollo de las fases focalizar e implantar.

\section{Conclusiones}

Dado que los servicios son cada vez más complejos y personalizados, y con ciclos de vida más cortos, el proceso de innovación de las organizaciones de servicios requiere tanto la mejora de sus actividades como el uso de más y mejores herramientas y técnicas para la gestión de la innovación. Este trabajo muestra la aplicabilidad del modelo de innovación desarrollado por la Fundación para la Innovación Tecnológica COTEC, propone el índice COTEC de desarrollo del proceso de innovación y determina su validez al comparar los resultados del índice propuesto con el valor correspondiente a la opinión general del estado de la innovación proporcionada por los entrevistados de las organizaciones estudiadas. 
Procesos de Innovación en las Organizaciones de Salud y Educación D’Alvano, Luigi y Hidalgo, Antonio

La primera conclusión del estudio es que las barreras y recursos disponibles bajo los cuales desarrollan sus procesos de innovación las organizaciones de salud y educación estudiadas, explican en gran medida la secuencia con la cual estas organizaciones han desarrollado las fases de sus procesos de innovación. Las principales barreras identificadas son la presencia de un entorno económico poco favorable, costos de innovación elevados junto a la disponibilidad de recursos y competencias por parte de los suplidores tecnológicos en el caso de hospitales o clínicas tipo A, y presupuestos limitados junto con la experticia de gerencia de proyecto en las universidades con carrera de ingeniería.

La segunda conclusión es que ambos tipos de organizaciones presentan un nivel medio de desarrollo para la mayoría de las fases de sus procesos de innovación y un nivel próximo a estructurado para la organización. Las organizaciones han dado prioridad al desarrollo de las fases que le permiten los recursos disponibles. En el caso de las organizaciones de salud, el desarrollo de la innovación está soportado por los recursos proporcionados por los proveedores a través de la capacitación y el financiamiento de los equipos. En el caso de las organizaciones de educación está soportado en que los limitados recursos presupuestarios llevan a la focalización y el uso eficiente de las competencias de gerencia de proyectos durante la fase de implementación.

La tercera conclusión hace referencia a que en ambos tipos de organización, la fase menos desarrollada es vigilar, con lo que se corre el peligro que los proyec- tos seleccionados respondan más a los criterios e intereses de los grupos de investigación, en lugar de las exigencias de la sociedad asociadas a las tendencias tecnológicas, las nuevas exigencias regulatorias y los requerimientos presentes en el entorno que rodea dichas organizaciones de servicio. Por tanto, parece deseable que las organizaciones presten especial importancia al desarrollo de la fase vigilar con la finalidad de alinear sus procesos de investigación, desarrollo e innovación con las tendencias del entorno tecnológico, económico y social, en una línea de acción más orientada por las necesidades de sus consumidores, proveedores y la sociedad.

Finalmente, esta investigación se considera el punto de partida para próximos estudios que deberían detallar para cada fase del proceso de innovación, las variables específicas correspondientes a las prácticas, herramientas, técnicas y sistemas utilizados en el desarrollo de los proyectos.

\section{Referencias bibliográficas}

Arzola, Minerva y Mejías, Agustín (2007). Modelo conceptual para gestionar la innovación en empresas del sector servicios. Revista Venezolana de Gerencia. Vol. 12, No. 37, pp. 80-98.

Banco Central de Venezuela (BCV). (2010). Información Estadística. Caracas, Venezuela. http://www.bcv.gob.ve 10/10/2010.

Chen, Yubo y Xie, Jinhong (2005). Third-Party Product Review and Firm Marketing Strategy. Marketing Science, Vol. 24, No. 2, pp. 218-240.

Chiesa, Vittorio; Coughlan, Paul y Voss, Chriss (1996). Development of a technical in- 
novation audit. Journal of Product Innovation Management. Vol. 13, pp. 105-136.

Christensen, Clayton M. (1997). The Innovator's Dilemma. Harvard Business School Press. Boston. United States of America.

Den Hertog, Pim; Broersma, Lourens y Van Ark, Bart (2003). On the soft side of innovation: services innovation and its policy implications. De Economist. Vol. 151, pp. 4-403.

Den Hertog, Pim; Van der Aa, Wietze y de Jong, Mark (2010). Capabilities for managing service innovation: towards a conceptual framework. Journal of Service Management. Vol. 21, No. 4, pp. $490-514$

European Foundation for Quality Management (EFQM) (2003). Modelo EFQM de Excelencia. EFQM. Bruselas, Bélgica.

Fitzsimmons, James and Fitzsimmons, Mona (1998). Service Management: Operations, Strategy, and Information Technology. Irwin/McGraw-Hill. United States of America.

Freeman, Christopher (1974). The Economics of Industrial Innovation. Penguin Books. London. United Kingdom

Fundación para la innovación tecnológica Cotec (1999). Pautas Metodológicas en Gestión de la Tecnología y de la Innovación para Empresas TEMAGUIDE. Tomo 1: Introducción, y Módulo I: Perspectiva Empresarial. Cotec. Madrid. España.

Fundación para la innovación tecnológica COTEC (2004). Análisis del Proceso de Innovación en las Empresas de Servicio. COTEC. Madrid. España.

Gadrey, Jean (1992). L' Economie des Services. Reperes, La Decouverte, Paris. Francia.

Gadrey, Jean; Gallouj, Faiz y Weinstein, Olivier (1995). New modes of innova- tion. How services benefit industry. International Journal of Service Industry Management. Vol. 6, No. 3, pp. 4-16.

Goffin, Keith y Pfeiffer, Rolf (1999). Innovation management in UK and German Manufacturing Companies. AngloGerman Foundation Report Series, December. London. United Kingdom.

Hernández Sampieri, R.; Fernández Collado, C. y Baptista Lucio, P. (1997). Metodología de Investigación, McGraw Hill, Madrid. España.

Hidalgo, Antonio; León, Gonzalo y Pavón, Julián (2002). La Gestión de la Innovación y la Tecnología en las Organizaciones. Ediciones Pirámide, Madrid. España.

IFM and IBM. (2008). Succeeding through service innovation: A service perspective for education, research, business and government. University of Cambridge. Institute for Manufacturing. Cambridge. United Kingdom.

Karniouchina, Ekaterina; Victorino, Liana y Verma, Rohit (2005). Product and Service Innovation: ideas for future cross-disciplinary research. Journal of Product Innovation Management. Vol. 23, pp. 274-280.

Liikanen, Erkki (2003). A more Innovative, Entrepreneurial Europe. Opening address at the XI Congress of Eurochambres. Rome. 9 October. Italy.

National Institute of Standards and Technology (NIST) (2006). Baldrige National Quality Program Criteria for Performance Excellence. Http: //www. Baldrige.nist.gov (07/04/2007).

Organización para la Cooperación y el Desarrollo Económico (OCDE) (2006). Manual de Oslo. Guía para la recogida e interpretación de datos sobre innovación. Tercera edición, versión en español, Grupo Tragsa, España. 
Procesos de Innovación en las Organizaciones de Salud y Educación

D’Alvano, Luigi y Hidalgo, Antonio

Oke, Adegoke y Goffin, Keith (2001). Innovation management in the service sector. Management Focus, Summer issue.

Palop, Fernando y Vicente, José M. (1999). Vigilancia tecnológica e inteligencia competitiva. Su potencialidad para la empresa española. Fundación COTEC. Madrid. España.

Robinson, Terry; Clarke-Hill, Colin M. y Clarkson, Richard (2002). Differentiation through service: A perspective from the commodity chemicals sector. The Service Industries Journal, Vol. 22, No 3, pp. 149-166.

Schumpeter, Joseph (1934). The Theory of Economic Development. Harvard
University Press. Cambridge. Massachusetts. United States of America.

Sundbo, Jon (1997). Management of innovation in services. The Services Industries Journal. Vol. 17, No. 3, pp. 432-455.

Tidd, Joe; Bessant John y Pavitt Keith (2005). Managing Innovation: Integrating Technological, Market and Organizational Change. John Wiley \& Sons Chichester, England.

Toivonen, Marja y Tuominen, Tiina (2009). Emergence of innovations in services. The Services Industries Journal. Vol. 29, No. 7, pp. 887-902.

World Bank (2010). World Development Indicators 2010. Washington DC. United States of America. 